\title{
Wearable cameras can reduce dietary under-reporting: doubly labelled water validation of a camera-assisted $24 \mathrm{~h}$ recall
}

\author{
Luke Gemming $^{1 *}$, Elaine Rush ${ }^{2}$, Ralph Maddison ${ }^{1}$, Aiden Doherty ${ }^{3}$, Nicholas Gant ${ }^{4}$, Jennifer Utter ${ }^{5}$ \\ and Cliona Ni Mhurchu ${ }^{1}$ \\ ${ }^{1}$ National Institute for Health Innovation, University of Auckland, School of Population Health, Private Bag 92019, \\ Auckland Mail Centre, Auckland 1142, New Zealand \\ ${ }^{2}$ Sport and Recreation, Auckland University of Technology, Private Bag 92006, Auckland 1142, New Zealand \\ ${ }^{3}$ Nuffield Department of Population Health, University of Oxford, Old Road Campus, Oxford OX3 7LF, UK \\ ${ }^{4}$ Department of Sport and Exercise Science, Faculty of Science, University of Auckland, Auckland, New Zealand \\ ${ }^{5}$ Section of Epidemiology and Biostatistics, School of Population Health, Private Bag 92019, Auckland 1142, New Zealand \\ (Submitted 5 April 2014 - Final revision received 18 August 2014 - Accepted 16 October 2014 - First published online 28 November 2014)
}

\begin{abstract}
Preliminary research has suggested that wearable cameras may reduce under-reporting of energy intake (EI) in self-reported dietary assessment. The aim of the present study was to test the validity of a wearable camera-assisted $24 \mathrm{~h}$ dietary recall against the doubly labelled water (DLW) technique. Total energy expenditure (TEE) was assessed over $15 \mathrm{~d}$ using the DLW protocol among forty adults ( $n 20$

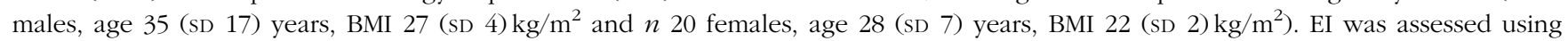
three multiple-pass $24 \mathrm{~h}$ dietary recalls (MP24) on days 2-4, 8-10 and 13-15. On the days before each nutrition assessment, participants wore an automated wearable camera (SenseCam (SC)) in free-living conditions. The wearable camera images were viewed by the participants following the completion of the dietary recall, and their changes in self-reported intakes were recorded (MP24+SC). TEE and EI assessed by the MP24 and MP24+SC methods were compared. Among men, the MP24 and MP24+SC measures underestimated TEE by 17 and $9 \%$, respectively $(P<0.001$ and $P=0.02)$. Among women, these measures underestimated TEE by 13 and $7 \%$, respectively $(P<0.001$ and $P=0.004)$. The assistance of the wearable camera (MP24+SC) reduced the magnitude of under-reporting by $8 \%$ for men and $6 \%$ for women compared with the MP24 alone $(P<0.001$ and $P<0.001)$. The increase in EI was predominantly from the addition of 265 unreported foods (often snacks) as revealed by the participants during the image review. Wearable cameras enhance the accuracy of self-report by providing passive and objective information regarding dietary intake. High-definition image sensors and increased imaging frequency may improve the accuracy further.
\end{abstract}

Key words: Nutrition assessment: Wearable cameras: SenseCam: Dietary studies

Traditional methods of dietary assessment are subjective and rely on self-report. Therefore, the accuracy of dietary data is influenced by memory recall, burden of administration ${ }^{(1)}$, psychosocial factors ${ }^{(2-4)}$, and other behavioural characteristics ${ }^{(5-8)}$, with $\operatorname{sex}(9-11)$, age $\mathrm{e}^{(11-14)}$, body $\operatorname{size} \mathrm{e}^{(11,14-18)}$ and ethnicity $^{(9-11,19)}$ all being shown to affect reporting.

To address these constraints, considerable interest has been shown in the use of image-assisted dietary assessment ${ }^{(20-24)}$. Handheld devices were first used to capture the images of foods manually, but the development of automated wearable cameras has allowed the capture of first-person point-of-view images to be explored ${ }^{(19,25-27)}$. Wearable cameras provide a new opportunity to improve the accuracy of dietary assessment as the images/videos provide a passive and objective record of an individual's eating episodes ${ }^{(21)}$.

Initial estimates suggest that wearable camera-assisted methods may have a relatively small measurement error for energy intake (EI) $(7 \%)^{(26)}$, and that they increase selfreported EI by 10-18\% compared with traditional methods $(19,27)$. The increased EI results from the detection of unreported foods, changes in reported portion size, and other misreporting errors identified within the images ${ }^{(19)}$. However, previous studies have relied on participants to manually capture images ${ }^{(26)}$, had small sample sizes $^{(19,26)}$, and none

Abbreviations: DLW, doubly labelled water; EI, energy intake; MP24, multiple-pass 24 h dietary recall; REE, resting energy expenditure; SC, SenseCam; TEE, total energy expenditure.

*Corresponding author: L. Gemming, fax +649373 1710, email l.gemming@auckland.ac.nz 
has validated wearable camera-assisted dietary assessment against a criterion measure, such as doubly labelled water (DLW) $^{(19,27)}$.

Therefore, the primary aim of the present study was to use the DLW technique to validate a wearable camera-assisted $24 \mathrm{~h}$ dietary recall. The analyses compared reported EI measured from $24 \mathrm{~h}$ dietary recalls alone and dietary recalls plus the wearable camera (SenseCam (SC)), and with total energy expenditure (TEE) estimated using the DLW method. A secondary objective was to examine the mechanism by which camera images enhance dietary recalls by quantifying alterations in self-report after viewing the images.

\section{Subjects and methods}

A total of forty volunteers, aged 18-64 years (twenty males and twenty females), from the greater Auckland city metropolitan area, New Zealand were recruited by advertisements on community notice boards located at fifteen supermarkets and three university campuses, and by a campaign through a participant recruitment service website (http://www. researchstudies.co.nz). The recruitment service sent email announcements to individuals who had previously indicated interest in human research, and used paid advertisement campaigns on Facebook. Potential participants who indicated interest were phoned, assessed for eligibility, and provided with written and verbal information regarding study procedures. Written informed consent was obtained from all the participants before the commencement of the study.

Participants were informed that the purpose of the research was to evaluate the use of Passive Image Capture to Record Everyday Events using wearable cameras. There was no specific reference to a validation study, but participants were informed that the images would be used to help assess their dietary intake and other health behaviours, such as the time spent watching television or travelling to work (not reported here).

All eligible participants were in self-reported good health, were not actively pursuing weight loss, and did not plan to conduct additional physical activity (above normal) or travel during the study period. Pregnant and lactating females, or individuals with a recent acute illness, who had their blood tests taken or took intravenous fluids 2 weeks before the study period were excluded. Recruitment efforts were targeted at all adults aged 18-65 years; however, it was ensured that an equal number of male and female participants were selected for the study. The University of Auckland Human Participants Ethics Committee approved the research (reference no. 8701). An $\$ 80$ New Zealand Dollar gift card was given to the participants at the completion of the study to compensate them for their time.

\section{Study design}

The present study was a cross-sectional, repeated-measures design. Each participant took part in the study over $15 \mathrm{~d}$ in free-living conditions and wore a wearable camera for $4 \mathrm{~d}$, and data were collected at four appointments (a baseline assessment and three follow-up dietary assessments). Data collection was conducted between March and September 2013.

Participants were contacted $1-3 \mathrm{~d}$ before their scheduled baseline assessment to reaffirm the study procedures and to schedule the nutrition assessments. Participants fasted overnight $(\geq 10 \mathrm{~h})$ and were instructed to refrain from any strenuous activity the day before the baseline assessment. Anthropometric characteristics including weight in light clothing with shoes and jewellery removed $( \pm 0.05 \mathrm{~kg}$, Tanita BWB-620), height $( \pm 0 \cdot 1 \mathrm{~cm}$, Seca 213), and percentage of body fat ( $\pm 5 \mathrm{ohm}$, Imp DF50; ImpediMed) were assessed.

Resting energy expenditure (REE) was measured before the participants were given a weight-specific dose of DLW to determine TEE during the study period. Participants then wore a wearable camera (SC) for $4 \mathrm{~d}$ : one familiarisation day; each day before three interviewer-administered multiple-pass $24 \mathrm{~h}$ dietary recalls (MP24) conducted on days 2-4 (nutrition assessment 1), days 8-10 (nutrition assessment 2) and days 13-15 (nutrition assessment 3). Therefore, there was a minimum of $3 \mathrm{~d}$ and a potential maximum of $8 \mathrm{~d}$ between the nutrition assessments.

For the determination of TEE, five timed urine samples were collected at baseline, $5 \mathrm{~h}$ post-dose, and on days 3, 9 and 15 . Participants were instructed to collect at least $50 \mathrm{ml}$ of the second void of the day, to place and seal the sample in a specimen pot, and to record the time of void on a form provided. The samples were collected from the participants at the earliest opportunity, either at the next nutrition assessment or from their home (if diet was assessed before the collection). To assess any weight change during the testing period, body mass was reassessed on day 15 using the same scales. During the study period, participants were told to follow their usual daily routine. A basic instruction leaflet for SC and a timeline of scheduled study assessments were provided at the end of the baseline assessment. Standardised text messages were used to remind the participants of the study protocol (e.g. day 3: please remember to collect urine sample \#3 (not the first void of the day) Passive Image Capture to Record Everyday Events study). Messages were sent at times to align with the participant's reported usual daily schedule.

\section{Use of the wearable camera}

SC is a wearable camera worn around the neck on a lanyard with a wide-angled lens ${ }^{(28,29)}$. Sensors detect movement (accelerometer), heat (IR) and light to trigger image capture approximately every $20 \mathrm{~s}$ (approximately 2000-3000 images/ d). Internal flash memory is sufficient for 1 week and battery capacity is adequate for a typical $12-16 \mathrm{~h} / \mathrm{d}$. Once turned on, SC operates continuously until the camera is switched off, or a privacy button can be activated to cease image capture temporally $(7 \mathrm{~min})$. Participants were provided instructions to operate and wear the device correctly at the baseline assessment. On the recording days, participants were instructed to wear the device from wake time (after bathing and dressing) until bedtime, but they could remove the camera anytime they felt uncomfortable or in locations where photography was inappropriate (e.g. gymnasium or public restroom). 
An information sheet was provided to help the participants determine when the camera should be switched off or not be worn. The images were encrypted to ensure that participants could not view the images before the nutrition assessment, or if lost, they could not be viewed by third parties. After the completion of each MP24, participants were provided with a chance to screen the images privately and instructed to delete any image they did not wish to disclose before a joint image review with the researcher ${ }^{(30)}$.

\section{Energy intake assessment}

Multiple-pass $24 \mathrm{~h}$ dietary recalls. Dietary recalls were conducted by a trained dietitian (L. G.) using a pen-andpaper-based multiple-pass method with a forgotten foods list to probe for unreported foods, adopted from the US Department of Agriculture ${ }^{(31)}$ followed by an image review (see Fig. 1). The assessments were conducted at the University of Auckland or at participants' homes/workplaces. Standard household measures, example crockery and glassware, and a portion size guide were used to assist the participants to estimate portion sizes. The portion size guide used ${ }^{(32)}$ was developed for the Australian population where the food supply is similar to that in New Zealand (no New Zealandspecific portion size guide was developed).

Multiple-pass $24 \mathrm{~h}$ dietary recalls + SenseCam. After the final pass of MP24, the researcher (L. G.) used Doherty's wearable camera browser ${ }^{(33)}$ to review the SC images with the participants (after the images were screened privately by the participants). The participants were instructed to confirm, modify, add or remove food items present in the images (MP24+SC). To assist the process, the researcher simply restated the foods and portions that were self-reported in

STEP

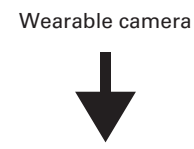

Nutrition assessment

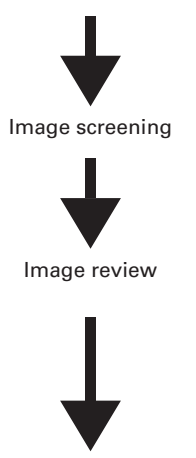

Alterations
TASK

Wearable camera worn before the dietary recalls to passively record eating episodes

Interviewer administered multiple pass $24 \mathrm{~h}$ dietary recall

Participants screened the images privately and deleted any image they wished

Images viewed by the researcher in a time-lapse progression that was stopped when foods were present or when eating looking likely (e.g. making a sandwich in the kitchen or ordering a meal in the cafeteria)

Participants asked to confirm or modify self-report without suggestions from the researcher

Fig. 1. Procedure to estimate energy intake in wearable camera-assisted dietary recalls. the MP24, and queried any unreported food items present in the images, but did not suggest any changes or scrutinise self-reported intakes. All changes made by the participants were detailed to determine the frequency and impact on EI. All unreported foods, misreporting errors and alterations to portion size were grouped by the following food categories: breads and cereals; beverages (excluding water due to no energy content); fruit and vegetables; meats and fish; dairy products; snack foods (biscuits, sweets and other snack foods); condiments (spreads, sauces, dips and dressings); alcohol; and other. The changes in self-report were both individual foods and composite foods (some foods cannot be separated into individual components).

\section{Measurement of total energy expenditure}

Daily TEE was measured using the DLW method. At the baseline assessment, participants ingested a pre-mixed dose of approximately $0 \cdot 1 \mathrm{~g}$ of $99.9 \%{ }^{2} \mathrm{H}_{2} \mathrm{O} / \mathrm{kg}$ total body water and $2 \mathrm{~g}$ of $10 \% \mathrm{H}_{2}^{18} \mathrm{O} / \mathrm{kg}$ total body water. To ensure that the full dose was consumed, the dose bottle was rinsed three times with tap water followed by an additional mouth rinse. To ascertain background isotope levels, a baseline urine sample was collected before dosing. Participants collected timed urine samples ( $5 \mathrm{~h}$ post-dose and on days 3,9 and 15) that were frozen (in duplicate in glass bottles) until study completion and analysed using an elemental analyser (Thermo Scientific TC/EA) coupled with a isotope ratio mass spectrometer (Thermo Scientific DeltaV advantage). Daily TEE was calculated by the multi-point method using linear regression from the difference between the elimination constants of ${ }^{18} \mathrm{O}$ and ${ }^{2} \mathrm{H}$, with individual respiratory quotient determined using the mean of the three MP24+SC measures $^{(34)}$.

\section{Resting energy expenditure}

A standardised protocol was used to assess REE between 07.00 and 10.00 hours. TEE was divided by REE to calculate the participants' activity factor during the study period. Participants were instructed to fast overnight $(\geq 10 \mathrm{~h})$ and refrain from any strenuous activity the day before the assessment. Before commencement, participants were positioned (near supine) on a folding bed for $\geq 10 \mathrm{~min}$, and asked to relax but remain awake. Testing was conducted in an environmental chamber maintained at $22^{\circ} \mathrm{C}$ with lights turned off during all assessments (participants remained awake throughout). REE was measured by indirect calorimetry using a mouthpiece and nose clip, and analysed breath by breath with a Moxus Modular system (S-3A/I Oxygen Analyser, CD-3A Carbon Dioxide Analyser and KTC3 Turbine Volumetric System; AEI Technologies). A $30 \mathrm{~min}$ measurement protocol was followed, with the data from the first $10 \mathrm{~min}$ and final 2 min being omitted, along with the periods of movement by the participants. Before each assessment, the metabolic cart was calibrated with standard gas mixtures and a volumetric syringe. 


\section{Statistical analysis}

Data of all participants were included in the final analysis, as device non-compliance and technical issues best reflect free-living conditions. Dietary intake was analysed using the nutrient analysis software FoodWorks 7 Professional edition (Xyris Software). Data analysis was performed using the software package for statistical analysis SPSS Statistics (version 20.0; IBM). Paired $t$ tests were used to compare differences in self-reported EI between the MP24 and MP24+SC methods used, and between EI and TEE. Limits of agreement between self-reported EI measured from the MP24 and MP24+SC methods, and TEE were assessed according to the recommendations of Bland \& Altman $^{(35)}$. Alterations in self-report (MP24+SC) and participant characteristics were described by summary statistics. Statistical significance was set at $\alpha \leq 0.05$.

\section{Results}

All the participants completed the study procedures. The general characteristics of the study population are presented in Table 1. Participants were predominantly New Zealand Europeans and the majority had tertiary education; however, differences in age, body size and education between the male and female participants were apparent. Men were predominately overweight or obese (65\%) and approximately 8 years older than women who were generally of normal body weight $(75 \%)$. Moreover, a greater proportion of women had attended university or completed graduate degrees ( $80 v .65 \%$ ). The mean body weight did not differ significantly between day 0 and day 15 for men or women (mean difference -0.1 (sD 0.9) kg, $P=0.546$ and -0.1 (sD 1.0$) \mathrm{kg}$, $P=0 \cdot 716$, respectively).

TEE measures and EI data for all the forty participants were used. Only three participants did not wear the camera for one of the three recording days (two participants reported that

Table 1. Demographic characteristics of the study participants

(Mean values and standard deviations)

\begin{tabular}{|c|c|c|c|c|}
\hline & \multicolumn{2}{|c|}{ Male } & \multicolumn{2}{|c|}{ Female } \\
\hline & Mean & SD & Mean & SD \\
\hline Age (years) & 34.8 & $12 \cdot 6$ & $27 \cdot 1$ & 7.5 \\
\hline Weight (kg) & $86 \cdot 3$ & $14 \cdot 7$ & $61 \cdot 3$ & $9 \cdot 7$ \\
\hline Height (m) & 178.0 & $6 \cdot 1$ & $165 \cdot 3$ & $6 \cdot 9$ \\
\hline \multirow[t]{2}{*}{$\mathrm{BMI}\left(\mathrm{kg} / \mathrm{m}^{2}\right)$} & $27 \cdot 1$ & 3.9 & $22 \cdot 3$ & $2 \cdot 3$ \\
\hline & $n$ & $\%$ & $n$ & $\%$ \\
\hline \multicolumn{5}{|l|}{ Body size } \\
\hline Normal & 7 & 35 & 17 & 85 \\
\hline Overweight & 9 & 45 & 3 & 15 \\
\hline Obese & 4 & 20 & 0 & 0 \\
\hline \multicolumn{5}{|l|}{ Ethnicity } \\
\hline New Zealand European & 14 & 70 & 15 & 75 \\
\hline Maori & 2 & 10 & 1 & 5 \\
\hline Asian & 4 & 20 & 4 & 20 \\
\hline \multicolumn{5}{|l|}{ Education } \\
\hline High school or less & 7 & 35 & 4 & 20 \\
\hline $\begin{array}{l}\text { University diploma or } \\
\text { undergraduate degree }\end{array}$ & 10 & 50 & 11 & 55 \\
\hline Postgraduate degree & 3 & 15 & 5 & 25 \\
\hline
\end{tabular}

Table 2. Distribution of the dietary recalls collected for the total sample of participants* by day of the week

\begin{tabular}{lcc}
\hline & Male (\%) & Female (\%) \\
\hline Monday & 18 & 12 \\
Tuesday & 15 & 13 \\
Wednesday & 18 & 22 \\
Thursday & 12 & 22 \\
Friday & 15 & 12 \\
Saturday & 10 & 8 \\
Sunday & 12 & 12 \\
\hline "Each participant $(n$ 40) had three multiple-pass dietary recalls \\
$\quad$ obtained over a 2-week period.
\end{tabular}

they were in bed for most of the day, and one participant was non-compliant for one of the three recording days), and six devices malfunctioned, which resulted in failure to capture the images. Additionally, on two occasions, participants forgot to bring the camera to the assessment venue. However, the MP24 was still conducted as usual and thus the MP24 and MP24+SC simply had the same values for these eleven instances ( $9 \%$ of the total dietary recalls). Regarding the image screening, the proportion of the participants that chose not to screen the images privately increased at each successive nutrition assessment ( $n$ 13, $n 18$ and $n$ 25, respectively). The median time of image screening was $5.5 \mathrm{~min}$ (range $1 \cdot 0-17 \cdot 1 \mathrm{~min}$, interquartile range $5 \cdot 2 \mathrm{~min}$ ).

For both males and females, the $24 \mathrm{~h}$ dietary recalls were distributed across all the days of the week, but the proportion was lower on weekend days, as shown in Table 2. The mean TEE measured by the DLW method and reported EI assessed from the three $24 \mathrm{~h}$ dietary recalls (MP24 and MP24+SC) are presented in Table 3. For men, the mean reported EI were 17\% (MP24) and 9\% (MP24+SC) below the measured TEE. For women, the mean reported EI were 13\% (MP24) and $7 \%$ (MP24+SC) below the TEE. The raw correlations between TEE and EI assessed by the MP24 and MP24+SC methods were, respectively, 0.68 and 0.61 for men. The correlations were, respectively, 0.82 and 0.81 for women. An assessment of agreement revealed that EI does not influence the magnitude of measurement error (see online supplementary material). The reduced magnitude of under-reporting of EI is presented in Fig. 2. The assistance of the wearable camera (MP24+SC) significantly reduced under-reporting for both men and women compared with the MP24 alone in all the three dietary recalls, and there was no significant difference observed between TEE and EI measured by the MP24+SC method in nutrition assessment 2.

Increased EI associated with the MP24+SC measure is presented in Fig. 3. There was no relationship evident between the increase in EI and TEE, but viewing the images sometimes resulted in a decreased EI. Alterations in the participants' selfreported EI are summarised in Table 4. The increase in reported EI was predominantly due to the addition of 265 unreported foods. Portion size was increased in most instances ( $n$ 49/51), but, overall, this had a less impact on reported EI than unreported foods that were removed or exchanged during the image review. 
Table 3. Daily energy intake (EI) measured in three multiple-pass $24 \mathrm{~h}$ dietary recalls (MP24 and MP24+SenseCam (SC)) and total energy expenditure (TEE) measured with the doubly labelled water technique

(Mean values and standard deviations)

\begin{tabular}{|c|c|c|c|c|c|c|c|c|c|c|c|c|}
\hline & \multicolumn{6}{|c|}{ Male $(n 20)$} & \multicolumn{6}{|c|}{ Female $(n 20)$} \\
\hline & \multicolumn{2}{|c|}{ El $(k J / d)$} & \multicolumn{2}{|c|}{ TEE (kJ/d) } & \multirow[b]{2}{*}{ TEE - El (\%) } & \multirow[b]{2}{*}{$P$} & \multicolumn{2}{|c|}{ El $(k J / d)$} & \multicolumn{2}{|c|}{ TEE (kJ/d) } & \multirow[b]{2}{*}{ TEE - El (\%) } & \multirow[b]{2}{*}{$P$} \\
\hline & Mean & SD & Mean & SD & & & Mean & SD & Mean & SD & & \\
\hline \multicolumn{13}{|l|}{ Total } \\
\hline MP24 & 12004 & 2122 & 14485 & 2632 & -17 & $<0.001$ & 9420 & 1694 & 10841 & 1639 & -13 & $<0.001$ \\
\hline MP24+SC & $13196^{\star}$ & 2529 & & & -9 & 0.02 & $10091^{*}$ & 1672 & & & -7 & 0.004 \\
\hline \multicolumn{13}{|l|}{ Recall 1} \\
\hline MP24 & 11770 & 3564 & 14485 & 2632 & -19 & 0.003 & 9253 & 1501 & 10841 & 1639 & -15 & $<0.001$ \\
\hline MP24+SC & $12543^{*}$ & 3941 & & & -13 & 0.02 & $9975^{\star}$ & 1674 & & & -8 & 0.02 \\
\hline \multicolumn{13}{|l|}{ Recall 2} \\
\hline MP24 & 12769 & 3183 & 14485 & 2632 & -12 & 0.008 & 9805 & 2699 & 10841 & 1639 & -10 & 0.013 \\
\hline MP24+SC & $14411^{*}$ & 3417 & & & -1 & 0.923 & $10455^{\star}$ & 2621 & & & -4 & 0.319 \\
\hline \multicolumn{13}{|l|}{ Recall 3} \\
\hline MP24 & 11472 & 3447 & 14485 & 2632 & -21 & 0.001 & 9202 & 2068 & 10841 & 1639 & -15 & $<0.001$ \\
\hline $\mathrm{MP} 24+\mathrm{SC}$ & $12634^{*}$ & 3331 & & & -13 & 0.025 & $9843^{*}$ & 1983 & & & -9 & 0.218 \\
\hline
\end{tabular}

${ }^{*}$ Mean value was significantly different from that of the MP24 $(P \leq 0.05)$.

REE data of one male and three females were excluded as the participants did not achieve a rested state during the REE procedures. REE and activity factor were, respectively, $7807(\mathrm{SD} 2125) \mathrm{kJ}$ and 1.9 (SD 0.5) for men and 6548 (SD 2033) $\mathrm{kJ}$ and 1.8 (SD 0.6) for women.

\section{Discussion}

The present study validated a wearable camera-assisted dietary recall against the DLW technique, and examined the impact of wearable cameras on dietary under-reporting. Overall, the assistance of the wearable cameras significantly reduced the magnitude of under-reporting of dietary EI by $8 \%$ for men and $6 \%$ for women compared with the dietary recall alone. The findings confirm the preliminary research suggesting that wearable cameras may reduce measurement error by revealing unreported foods and misreporting errors $^{(19,27)}$.

The degree of under-reporting of dietary EI observed in the MP24 alone (male 17\% and female 13\%) was similar to that reported in other studies on the DLW validation of $24 \mathrm{~h}$ dietary recalls, which have reported EI values approximately $8-24 \%$ below the $\mathrm{TEE}^{(36)}$. To date, when compared with the DLW study with the largest size of samples ( $n$ 524), which validated the automated multiple-pass $24 \mathrm{~h}$ dietary recall method, the level of under-reporting bias for dietary EI was comparable for males (14\% among overweight men and 20\% among obese men compared with $17 \%$ among men in the present study) but higher for females ( $6 \%$ among women with a normal body weight compared with $13 \%$ among women in the National Health and Nutrition Examination Survey) ${ }^{(31)}$. The lower value observed in the validation of the automated multiple-pass method was likely attributable to the use of the structured automated multiple-pass method software (not the pen-and-paper-based), and robust trial conditions used to replicate the procedures of the National Health and Nutrition Examination Survey ${ }^{(31)}$ in conjunction with a motivated sample, which received substantial financial incentives.
There have been only a few studies on the DLW validation of image-assisted dietary assessment methods. A custom-built wearable camera 'eButton' designed to objectively assess dietary intake and physical activity has been demonstrated, but is yet to be validated ${ }^{(20,21)}$. Other image-assisted dietary assessment methods in development differ as they require the participants to capture the images actively using smart phones or other handheld devices. Nonetheless, validation of the remote photography food method (a manually triggered image-based dietary record) against the DLW technique among free-living adults (predominantly overweight and obese) revealed reduced measurement error compared with traditional methods (mean EI 6\% below the TEE $v$. $12-49 \%$ among overweight and obese populations reported elsewhere $)^{(15-17,22,31,37-39)}$. Similar image-based

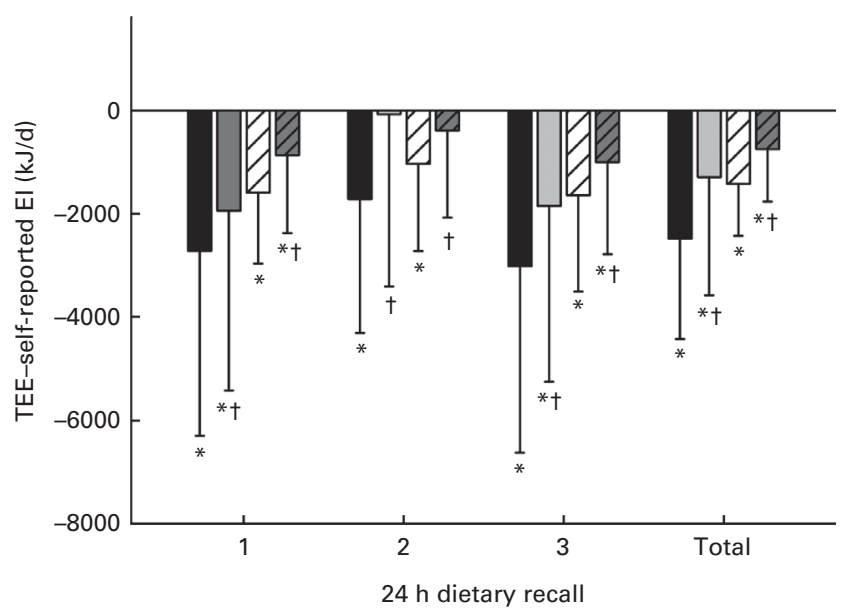

Fig. 2. Under-reported energy intake in the multiple-pass $24 \mathrm{~h}$ dietary recall alone (MP24) and with the assistance of the wearable camera (MP24+ SenseCam (SC)) compared with total energy expenditure (TEE) for men and women. * Mean value was significantly different from that of TEE $(P \leq 0.05)$. † Mean value was significantly different from that of the MP24 $(P \leq 0.05)$. MP24: (male), $\square$ (female); MP24+SC: $\square$ (male), $\square$ (female). 


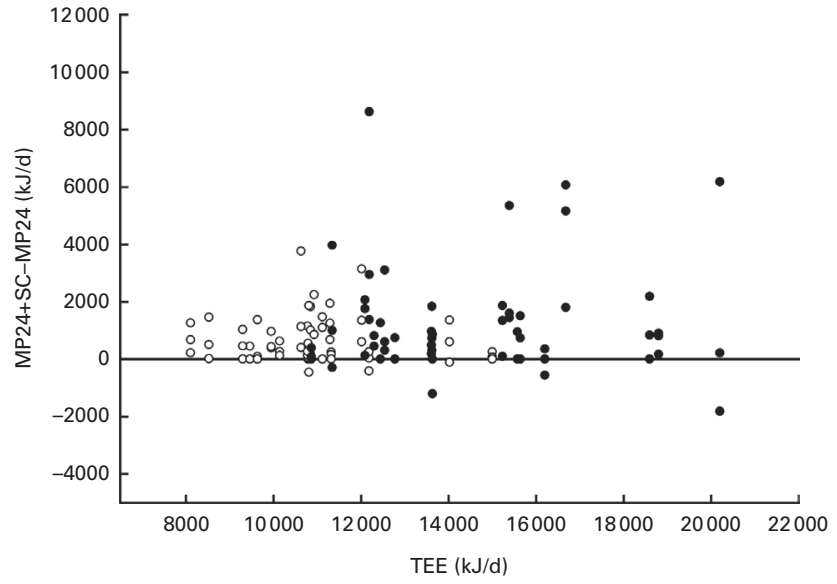

Fig. 3. Changes in self-reported energy intake in the multiple-pass $24 \mathrm{~h}$ dietary recalls (MP24) after viewing the wearable camera images (MP24+ SenseCam (SC)) compared with total energy expenditure (TEE) for men (•) and women (O) ( $n$ 120, three $24 \mathrm{~h}$ dietary recalls for each participant).

dietary records in development are yet to be validated with human participants in free-living settings ${ }^{(23,24)}$.

Several factors may explain why dietary under-reporting was not completely eliminated with the assistance of the wearable camera. The imaging frequency of wearable cameras was insufficient (two to three images per min) to capture all the foods consumed, and image quality was relatively poor, especially in low-light environments. Moreover, the position of the camera on the body allows the lens angle to be affected by posture, and foods in bowls or on high tables can be obscured easily. Intentional under-reporting may also go undetected if participants delete the images of foods during the private screening. However, the short duration that participants took to screen the images and the proportion of the participants who chose not to screen their images in the second and third nutrition assessments suggested this was not a frequent issue. Reactivity could also be a factor. Previous studies have indicated that the use of wearable cameras is a low burden, but may have an impact on the participants' usual dietary behaviours; however, the degree of behaviour change was unclear ${ }^{(19,26)}$. Additional dietary recalls for non-camera days would have provided a within-person comparison to assess reactivity.

Other study limitations include the relatively small heterogeneous sample that was not representative of the general population; therefore, the study may have produced different results. Moreover, due to differences in body size, male and female data were treated separately. However, participants of all body sizes were deliberately recruited as the earlier feasibility study has revealed that camera-assisted recalls were helpful for all body types of people ${ }^{(19)}$. Additionally, the prevalence of under-reporting increased substantially in New Zealand's most recent adult nutrition survey among people with a normal body weight, as well as among overweight and obese individuals ${ }^{(40)}$. Furthermore, a sole dietitian conducted nutrition assessments and the image review process; thus, interviewer bias cannot be ruled out (interview procedures were not audited). Additionally, dietary intake data may not be representative of usual intake, due to the short duration of the study, the limited number of dietary recalls conducted, and the lower proportion of weekend days $v$. weekdays ${ }^{(41)}$.

A unique feature of the study was the detailed comparison between traditional self-report alone $v$. self-report assisted by the wearable camera. The design of the present study allowed the determinants of under-reporting to be identified, and confirmed that unreported snack foods, condiments and beverages are the primary source of under-reported EI in a free-living setting. Similar to the feasibility study ${ }^{(19)}$, a range of foods were revealed by the images, and included both healthy and unhealthy foods. These were generally individual food items, such as biscuits, peanut butter and soda, but included some composite foods, such as cheese burgers, vegetable soup and butter chicken. Interestingly, fruit and vegetables were unreported frequently ( $n$ 47/265 foods). Often these were snacks, such as bananas, apples, carrots and raisins, which further highlighted the difficulty for participants to remember the details of their snacking episodes during retrospective assessments. The alterations by participants to portion size may have produced correlated errors (as the true portion size remained unknown) ${ }^{(42-44)}$ but in most instances, the portion size was clearly incorrect

Table 4. Alterations in self-reported energy intake for all the participants viewing wearable camera images after completion of the multiplepass $24 \mathrm{~h}$ dietary recalls

(Number of participants, mean values and standard deviations)

\begin{tabular}{|c|c|c|c|c|c|c|c|c|c|c|c|c|}
\hline & \multicolumn{4}{|c|}{ Unreported foods } & \multicolumn{4}{|c|}{ Portion size } & \multicolumn{4}{|c|}{ Misreported foods } \\
\hline & \multicolumn{4}{|c|}{ Energy (kJ) } & \multicolumn{4}{|c|}{ Energy (kJ) } & \multicolumn{4}{|c|}{ Energy (kJ) } \\
\hline & $n$ & Mean & SD & Total & $n$ & Mean & SD & Total & $n$ & Mean & SD & Total \\
\hline Breads/cereals & 23 & 462 & 162 & 10634 & 16 & 462 & 306 & 7386 & 3 & -607 & 191 & -1821 \\
\hline Beverages & 40 & 308 & 182 & 12334 & 6 & 26 & 80 & 155 & 13 & -135 & 149 & -1754 \\
\hline Fruit/vegetables & 47 & 153 & 98 & 7187 & 10 & 43 & 56 & 434 & 8 & -130 & 107 & -1037 \\
\hline Meat/fish/eggs & 11 & 590 & 140 & 6494 & 3 & 88 & 22 & 264 & 2 & -402 & 211 & -804 \\
\hline Dairy & 18 & 616 & 181 & 11090 & 2 & 333 & 78 & 667 & 3 & -113 & 56 & -340 \\
\hline Snack foods & 64 & 571 & 425 & 36547 & 3 & 1140 & 411 & 3419 & 3 & -1112 & 331 & -3335 \\
\hline Condiments & 50 & 307 & 270 & 15343 & 7 & 181 & 35 & 1266 & 1 & -184 & - & -184 \\
\hline Alcohol & 7 & 712 & 130 & 4983 & 1 & 128 & - & 128 & 0 & - & - & - \\
\hline Other & 5 & 1191 & 203 & 5957 & 3 & -30 & 105 & -91 & 3 & -2526 & 793 & -7577 \\
\hline Total & 265 & 417 & 222 & 110570 & 51 & 267 & 166 & 13628 & 36 & -468 & 311 & -16852 \\
\hline
\end{tabular}


(e.g. two $v$. one slice of toast). The decision not to question or scrutinise the participants' self-report during the image review reduced the potential for interviewer bias $^{(45)}$, but trained image analysts or use of automated image-analysis techniques could enhance the method further ${ }^{(22,46)}$.

The strength of automated wearable cameras over handheld devices is their ability to capture images passively, which means they are potentially less intrusive during daily activities and may reduce participant burden. Wearable technologies can also collect physical activity data passively (using inbuilt accelerometers and global positioning system (GPS)), a key lifestyle consideration often overlooked when collecting dietary intake data ${ }^{(47,48)}$. Thus, wearable technologies have a greater potential than simply revealing unreported foods and misreporting errors, which needs to be explored.

\section{Conclusions}

The wearable camera significantly reduced the magnitude of under-reporting in the $24 \mathrm{~h}$ dietary recall by $9 \%$ for men and $6 \%$ for women, as the images revealed unreported foods and misreporting errors not captured by the traditional method alone. Wearable cameras with faster imaging frequencies, high-definition image sensors, and the use of automated image analysis techniques may enhance the method further. Additional research is needed in larger representative samples of the population. Future studies should explore the use of wearable cameras in different settings using a variety of image-assisted methods.

\section{Supplementary material}

To view supplementary material for this article, please visit http://dx.doi.org/10.1017/S0007114514003602

\section{Acknowledgements}

The present study was not supported by any external funding source.

The authors' responsibilities are as follows: L. G., C. N. M., E. R., R. M., A. D. and N. G. contributed to the study design; L. G. and C. N. M. were involved in the data collection and study management; L. G., E. R., R. M. and N. G. carried out the energy expenditure analyses; L. G., C. N. M., E. R., R. M., A. D., N. G. and J. U. interpreted the data; L. G. performed the statistical data analysis; L. G. wrote the manuscript; C. N. M., E. R., R. M., A. D., N. G. and J. U. edited the manuscript.

None of the authors had a personal or financial conflict of interest.

\section{References}

1. Thompson FE \& Subar A (2001) Dietary assessment methodology. In Nutrition in the Prevention and Treatment of Disease, pp. 3-30 [A Coulston and C Boushe, editors]. San Diego, CA: Academic Press.
2. Tooze JA, Subar AF, Thompson FE, et al. (2004) Psychosocial predictors of energy underreporting in a large doubly labeled water study. Am J Clin Nutr 79, 795-804.

3. Taren DL, Tobar M, Hill A, et al. (1999) The association of energy intake bias with psychological scores of women. Eur J Clin Nutr 53, 570-578.

4. Maurer J, Taren DL, Teixeira PJ, et al. (2006) The psychosocial and behavioral characteristics related to energy misreporting. Nutr Rev 64, 53-66.

5. Edwards JE, Lindeman AK, Mikesky AE, et al. (1993) Energy balance in highly trained female endurance runners. Med Sci Sports Exerc 25, 1398-1404.

6. Barnard J, Tapsell L, Davies P, et al. (2002) Relationship of high energy expenditure and variation in dietary intake with reporting accuracy on 7 day food records and diet histories in a group of healthy adult volunteers. Eur J Clin Nutr 56, 358-367.

7. Westerterp K, Saris W, Van Es M, et al. (1986) Use of the doubly labeled water technique in humans during heavy sustained exercise. J Appl Physiol 61, 2162-2167.

8. Hassapidou M \& Manstrantoni A (2001) Dietary intakes of elite female athletes in Greece. J Hum Nutr Diet 14, 391-396.

9. Tomoyasu NJ, Toth MJ \& Poehlman ET (2000) Misreporting of total energy intake in older African Americans. Int $J$ Obes Relat Metab Disord 24, 20-26.

10. Harrison GG, Galal OM, Ibrahim N, et al. (2000) Underreporting of food intake by dietary recall is not universal: a comparison of data from Egyptian and American women. J Nutr 130, 2049-2054.

11. Pikholz C, Swinburn B \& Metcalf P (2004) Under-reporting of energy intake in the 1997 National Nutrition Survey. $N Z$ Med J 117, U1079.

12. Briefel R, Sempos CT, McDowell M, et al. (1997) Dietary methods research in the third National Health and Nutrition Examination Survey: underreporting of energy intake. $\mathrm{Am} \mathrm{J}$ Clin Nutr 65, 1203S-1209S.

13. Johansson L, Solvoll K, Bjørneboe G-E, et al. (1998) Underand overreporting of energy intake related to weight status and lifestyle in a nationwide sample. Am J Clin Nutr $\mathbf{6 8}$, 266-274.

14. Tomoyasu NJ, Toth MJ \& Poehlman ET (1999) Misreporting of total energy intake in older men and women. J Am Geriatr Soc $\mathbf{4 7}, 710-715$.

15. Bandini LG, Schoeller DA, Cyr HN, et al. (1990) Validity of reported energy intake in obese and nonobese adolescents. Am J Clin Nutr 52, 421-425.

16. Bandini LG, Vu D, Must A, et al. (1999) Comparison of highcalorie, low-nutrient-dense food consumption among obese and non-obese adolescents. Obes Res 7, 438-443.

17. Lichtman SW, Pisarska K, Berman ER, et al. (1992) Discrepancy between self-reported and actual caloric intake and exercise in obese subjects. $N$ Engl J Med 327, 1893-1898.

18. Heerstrass D, Ocke M, Bueno-de-Mesquita H, et al. (1998) Underreporting of energy, protein and potassium intake in relation to body mass index. Int J Epidemiol 27, 186-193.

19. Gemming L, Doherty A, Kelly P, et al. (2013) Feasibility of a SenseCam-assisted 24-h recall to reduce under-reporting of energy intake. Eur J Clin Nutr 67, 1095-1099.

20. Bai Y, Li C, Yue Y, et al. (editors). (2012) Designing a wearable computer for lifestyle evaluation. In Proceedings of the 38th Annual Northeast Bioengineering Conference (NEBEC 2012). Philadelphia, PA: IEEE. 
21. Sun M, Fernstrom JD, Jia W, et al. (2010) A wearable electronic system for objective dietary assessment. I Am Diet Assoc 110, 45-47.

22. Martin CK, Correa JB, Han H, et al. (2012) Validity of the Remote Food Photography Method (RFPM) for estimating energy and nutrient intake in near real-time. Obesity (Silver Spring) 20, 891-899.

23. Weiss R, Stumbo PJ \& Divakaran A (2010) Automatic food documentation and volume computation using digital imaging and electronic transmission. J Am Diet Assoc 110, $42-44$.

24. Six BL, Schap TE, Kerr DA, et al. (2011) Evaluation of the Food and Nutrient Database for Dietary Studies for use with a mobile telephone food record. J Food Compost Anal 24, 1160-1167.

25. Arab L \& Winter A (2010) Automated camera-phone experience with the frequency of imaging necessary to capture diet. J Am Diet Assoc 110, 1238-1241.

26. Arab L, Estrin D, Kim DH, et al. (2011) Feasibility testing of an automated image-capture method to aid dietary recall. Eur J Clin Nutr 65, 1156-1162.

27. O'Loughlin G, Cullen SJ, McGoldrick A, et al. (2013) Using a wearable camera to increase the accuracy of dietary analysis. Am J Prev Med 44, 297-301.

28. Hodges S, Williams L, Berry E, et al. (2006) SenseCam: a retrospective memory aid. In UbiComp 2006: Ubiquitous Computing; 2006 Sep 17-21, pp. 177-193 [P Dourish and A Friday, editors]. Orange County, CA: Springer-Verlag.

29. Doherty AR, Hodges SE, King A, et al. (2012) Wearable cameras in health: the state of the art and future possibilities. Am J Prev Med 44, 320-323.

30. Kelly P, Marshall SJ, Badland H, et al. (2013) An ethical framework for automated, wearable cameras in health behavior research. Am J Prev Med 44, 314-319.

31. Moshfegh AJ, Rhodes DG, Baer DJ, et al. (2008) The US Department of Agriculture Automated Multiple-Pass Method reduces bias in the collection of energy intakes. Am J Clin Nutr 88, 324-332.

32. Probst Y (2012) Food Portion Images for Dietary Assessment: Graduated Food Portion Photos of Australian Foods. Wollongong: The University of Wollongong.

33. Doherty AR, Pauly-Takacs K, Caprani N, et al. (2012) Experiences of aiding autobiographical memory using the SenseCam. Int J Hum Comput Interact 27, 151-174.

34. Prentice A (1990) Doubly Labeled Water Method for Measuring Energy Expenditure: Technical Recommendations for Use in Humans. Vienna: International Atomic Energy Agency.
35. Bland J \& Altman D (1986) Statistical methods for assessing agreement between two methods of clinical measurement. Lancet 327, 307-310.

36. Livingstone MB \& Black AE (2003) Markers of the validity of reported energy intake. J Nutr 133, Suppl. 3, 895S-920S.

37. Buhl KM, Gallagher D, Hoy K, et al. (1995) Unexplained disturbance in body weight regulation: diagnostic outcome assessed by doubly labeled water and body composition analyses in obese patients reporting low energy intakes. J Am Diet Assoc 95, 1393-1400.

38. Goris AHC, Westerterp-Plantenga MS \& Westerterp KR (2000) Undereating and underrecording of habitual food intake in obese men: selective underreporting of fat intake. Am J Clin Nutr 71, 130-134.

39. Black AE, Jebb SA, Bingham SA, et al. (1995) The validation of energy and protein intakes by doubly labelled water and 24-hour urinary nitrogen excretion in post-obese subjects. J Hum Nutr Diet 8, 51-64.

40. Gemming L, Jiang Y, Swinburn B, et al. (2014) Underreporting remains a key limitation of self-reported dietary intake: an analysis of the 2008/09 New Zealand Adult Nutrition Survey. Eur J Clin Nutr 68, 259-264.

41. Basiotis PP, Welsh SO, Cronin FJ, et al. (1987) Number of days of food intake records required to estimate individual and group nutrient intakes with defined confidence. $J$ Nutr 117, 1638-1641.

42. Hernández T, Wilder L, Kuehn D, et al. (2006) Portion size estimation and expectation of accuracy. J Food Compost Anal 19, S14-S21.

43. Nelson M, Atkinson M \& Darbyshire S (1994) Food photography. I: the perception of food portion size from photographs. Br J Nutr 72, 649-663.

44. Ovaskainen M, Paturi M, Reinivuo H, et al. (2007) Accuracy in the estimation of food servings against the portions in food photographs. Eur J Clin Nutr 62, 674-681.

45. Gibson RS (2005) Measurement errors in dietary assessment. In Principles of Nutrition Assessment, 2nd ed., USA: Oxford University Press.

46. Zhu F, Bosch M, Boushey CJ, et al. (2010) An image analysis system for dietary assessment and evaluation. In Proceedings/ICIP International Conference on Image Processing, pp. 1853-1856. Hong Kong: IEE.

47. Black AE (2000) The sensitivity and specificity of the Goldberg cut-off for EI:BMR for identifying diet reports of poor validity. Eur I Clin Nutr 54, 395-404.

48. Black AE (2000) Critical evaluation of energy intake using the Goldberg cut-off for energy intake: basal metabolic rate. A practical guide to its calculation, use and limitations. Int J Obes Relat Metab Disord 24, 1119-1130. 